\title{
Prognostic value of programmed cell death-ligand I expression in patients with non-small-cell lung cancer: evidence from an updated meta-analysis
}

This article was published in the following Dove Press journal:

OncoTargets and Therapy

I December 2015

Number of times this article has been viewed

\author{
Anyuan Zhongl,* \\ Yufei Xingl,* \\ Xue $\operatorname{Pan}^{1, *}$ \\ Minhua Shi' \\ Huajun $\mathrm{Xu}^{2}$ \\ 'Department of Respiratory Diseases, \\ the Second Affiliated Hospital \\ of Soochow University, Suzhou, \\ ${ }^{2}$ Department of Otolaryngology, \\ Shanghai Jiao Tong University \\ Affiliated Sixth People's Hospital, \\ Otolaryngology Institute of Shanghai \\ Jiao Tong University, Shanghai, \\ People's Republic of China \\ *These authors contributed equally \\ to this work
}

Background: The association between the expression of programmed cell death-ligand 1 (PD-L1) and survival in patients with non-small-cell lung cancer (NSCLC) is controversial. Thus, we conducted a meta-analysis of all available studies to evaluate the prognostic role of PD-L1 expression in NSCLC.

Materials and methods: PubMed, Embase, and Chinese (China National Knowledge Infrastructure and Wanfang) databases were searched to identify all eligible studies evaluating PD-L1 expression and the survival of NSCLC patients. Hazard ratios (HRs) and 95\% confidence interval (CI) used to assess overall survival were extracted and pooled. Subgroup, sensitivity, and publication-bias analyses were also performed.

Results: Eleven articles reporting 12 studies that included a total of 1,653 patients met the inclusion criteria and were included in the meta-analysis. Higher PD-L1 expression did not correlate with prognosis in terms of overall survival in patients with NSCLC (HR $=1.21,95 \%$ CI: $0.85-1.71, P=0.29)$. However, a subgroup analysis showed a significant association between PD-L1 expression and poor prognosis in Chinese patients with NSCLC (HR $=1.55,95 \%$ CI: $1.04-2.29, P=0.03)$. The sensitivity analysis showed that the pooled results were not affected by the removal of any single study. There was also no significant publication bias.

Conclusion: Our meta-analysis indicated no statistically significant difference between PD-L1 expression and prognosis for patients with NSCLC. Additional, high-quality studies with larger sample sizes are needed to determine the prognostic value of PD-L1 expression in NSCLC.

Keywords: non-small-cell lung cancer, programmed cell death-ligand 1, prognosis, metaanalysis

\section{Introduction}

Lung cancer is the most commonly diagnosed cancer worldwide and the leading cause of cancer-related death. Non-small-cell lung cancer (NSCLC) accounts for $80 \%-85 \%$ all lung cancer cases. ${ }^{1}$ The majority of patients with NSCLC present with advanced disease. $^{2}$ Thus, despite improved forms of treatment (chemotherapy, radiotherapy, and surgery), the 5 -year survival rate is still $<15 \% .^{3}$ Tumor node metastasis stage, patient age, performance status, and weight loss have been identified as independent prognostic factors in NSCLC ${ }^{4}$ However, because the predictive power of these factors is unreliable, ${ }^{5,6}$ more broadly useful prognostic biomarkers are needed.

In the treatment of several kinds of cancer, including lung cancer, potential therapeutic targets such as epidermal growth factor receptor (EGFR) ${ }^{7}$ Kirsten rat sarcoma viral oncogene, ${ }^{8}$ and human EGFR- $2,{ }^{9}$ have been the focus of recent attention. Yet, the development of drugs aimed at these targets has been hampered by drug resistance and a high mutation rate of the relevant genes. 
The surface receptor programmed cell death 1 (PD-1) is a 288-amino acid cell surface protein and a member of the B7-CD28 superfamily. ${ }^{10}$ PD-1 is expressed on the surface of activated $\mathrm{T}$ and $\mathrm{B}$ cells and regulates their activation and proliferation. ${ }^{11}$ PD-ligand 1 (PD-L1) binds to the PD-1 receptor, leading to, among other responses, the negative regulation of immune activity. PD-L1 is also thought to be involved in the ability of cancer cells to evade host immune surveillance. ${ }^{12}$ For example, PD-L1 expressed on tumor cells was shown to promote apoptosis of antigen-specific and tumor-reactive T-cells, resulting in enhanced tumor cell growth. PD-L1 expression has been evaluated in a number of human cancers, including NSCLC, melanoma, esophageal adenocarcinoma, kidney tumors, and breast, bladder, ovarian, pancreatic, and esophageal cancers. ${ }^{13-17}$ However, studies on the prognostic value of PD-L1 expression in patients with NSCLC have yielded inconsistent results and have been limited by their low statistical power. ${ }^{18-28}$ To address these issues, we conducted a meta-analysis to determine the prognostic value of PD-L1 expression in patients with NSCLC.

\section{Materials and methods}

We performed this meta-analysis according to the guidelines of the Preferred Reporting Items for Systematic Reviews and Meta-analyses. ${ }^{29}$

\section{Literature search}

A comprehensive literature search was performed using the electronic databases PubMed, Embase, China National Knowledge Infrastructure, and Wanfang. The last search was performed on June 10, 2015. The search terms used were as follows: "Programmed cell death-ligand 1", "PD-L1", "B7-H1", "lung cancer", "survival", and "prognosis". All references cited in relevant articles were also checked to identify additional published work. No restrictions were applied during database searching, which was performed independently by two investigators.

\section{Inclusion and exclusion criteria}

Studies eligible for inclusion in this meta-analysis had to meet the following criteria: 1) the cancer was histologically confirmed as NSCLC; 2) PD-L1 expression was measured in lung cancer tissue using immunohistochemistry (IHC); 3 ) the association between PD-L1 expression and NSCLC prognosis was investigated; 4) sufficient data were available to estimate overall survival (OS) or associations between PD-L1 expression; and 5) PD-L1 expression was scored as "high (positive)" or "low (negative)". To avoid duplication of the data, only the most complete and recent of two related studies were included. Exclusion criteria were: 1) studies reported in reviews or letters, ongoing studies, and conference papers; 2) nonclinical studies and studies of other types of cancer; and 3) studies with insufficient survival data.

\section{Data extraction}

Two investigators (AZ and YX) extracted the following required data independently from all eligible studies: the first author's name, year of publication, country, cancer type, cancer stage, test method, cutoff value for positive PD-L1 expression, primary antibody, sample size, hazard ratio (HR) estimation method, and treatment. Discrepancies in data collection were resolved by group discussion. Questions were resolved, and details from a particular study were obtained by contacting the study authors via email.

\section{Statistical analysis}

All statistical analyses were performed using Stata software (v11.0; StataCorp LP, College Station, TX, USA). HRs with $95 \%$ confidence intervals (CIs) were used to assess the effect of high PD-L1 expression on the survival of patients with NSCLC. If these data were provided indirectly, the HRs and 95\% CIs were calculated according to Tierney's methods. ${ }^{30}$ Subgroup analyses were performed with respect to the geographic region of the patients, cancer type, HR estimation methods, primary antibody (source and type), and treatment method. Heterogeneity among studies was tested using the $\mathrm{Q}$ and $I^{2}$ statistics. For $P>0.10$, the Mantel-Haenszel fixedeffects model was used; otherwise, DerSimonian and Laird's random-effects model was used. ${ }^{31,32}$ A sensitivity analysis was performed by a leave-one-out procedure. ${ }^{33}$ Publication bias was evaluated using a funnel plot and objective Begg's or Egger's tests. ${ }^{34,35}$ Unless otherwise stated, $P<0.05$ was considered to be statistically significant.

\section{Results}

\section{Search results}

After searching the aforementioned electronic databases, 125 references were obtained. Of these, 33 references were excluded because of duplication, and another 35 (26 reviews, one letter, two ongoing studies, and six conference papers) were excluded after their titles and abstracts were read. After the full-text of the remaining articles was read, 22 nonclinical studies, five studies referring to other types of cancer, and 19 studies with insufficient survival data were further excluded. Thus, ultimately, eleven articles reporting on 12 studies were included in our meta-analysis and were subjected to further statistical evaluation (Figure 1). 


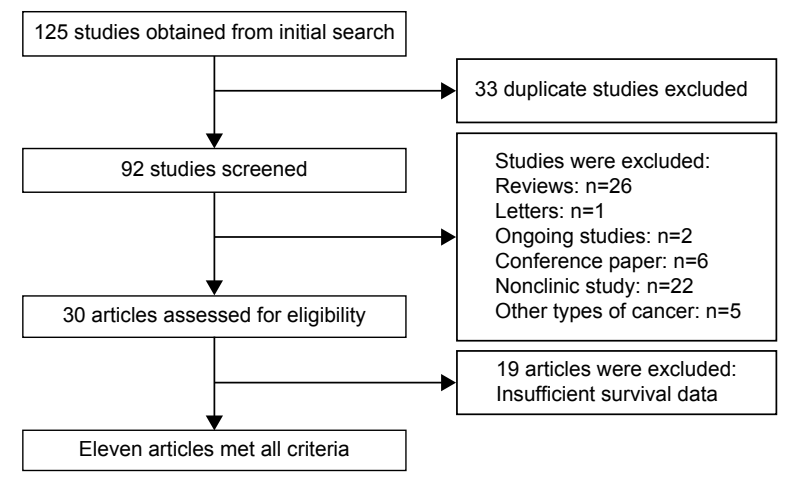

Figure I Flow chart of studies in the analysis.

\section{Study characteristics}

Table 1 summarizes the characteristics of the included studies. The year of publication ranged from 2010 to 2015. The 12 studies reported by the eleven articles included a total of 1,653 participants. Eight of the studies were performed in the People's Republic of China and the other four in Japan, Greece, the USA, and Italy (one each). The sample size of the included studies ranged from 47 to 340 . Five studies consisted of patients with stages I-III NSCLC, three studies included patients with stages IIIB-IV disease, and another three studies comprised patients with stages I-IV disease. With respect to the histological type of NSCLC, three studies evaluated patients with adenocarcinoma, and the others focused on NSCLC including adenocarcinoma, squamous cell cancer, and large cell cancer. All studies used IHC to evaluate PD-L1 expression in patients with NSCLC. In the four articles that did not report HRs and 95\% CIs directly, Kaplan-Meier curves were used to calculate these statistics.

\section{PD-LI expression and OS}

The pooled results of the 12 trials comprising 1,653 patients did not show a statistically significant relationship between PD-L1 expression and OS (HR $=1.21,95 \%$ CI: $0.85-1.71$, $P=0.29)$. However, there was significant between-study heterogeneity $\left(I^{2}=82 \%, P<0.001\right)$ (Figure 2$)$.

\section{Subgroup analysis between PD-LI expression and OS}

Subgroup analysis showed that PD-L1 expression was significantly associated with poor prognosis in Chinese patients (HR $=1.55,95 \%$ CI: $1.04-2.29, P=0.03)$. In the remaining subgroups, a statistical relationship between PD-L1 expression and NSCLC prognosis could not be determined. The results of the subgroup analyses are presented in Table 2 .

\section{Sensitivity analyses}

Sensitivity analysis was conducted by sequentially omitting each trial one at a time. The results showed that no single trial remarkably altered the pooled results for OS, suggesting they were robust and reliable (Figure 3).

\section{Publication bias}

According to Begg's test $(P=0.20)$ and Egger's test $(P=0.53)$, there was no significant publication bias for any of the

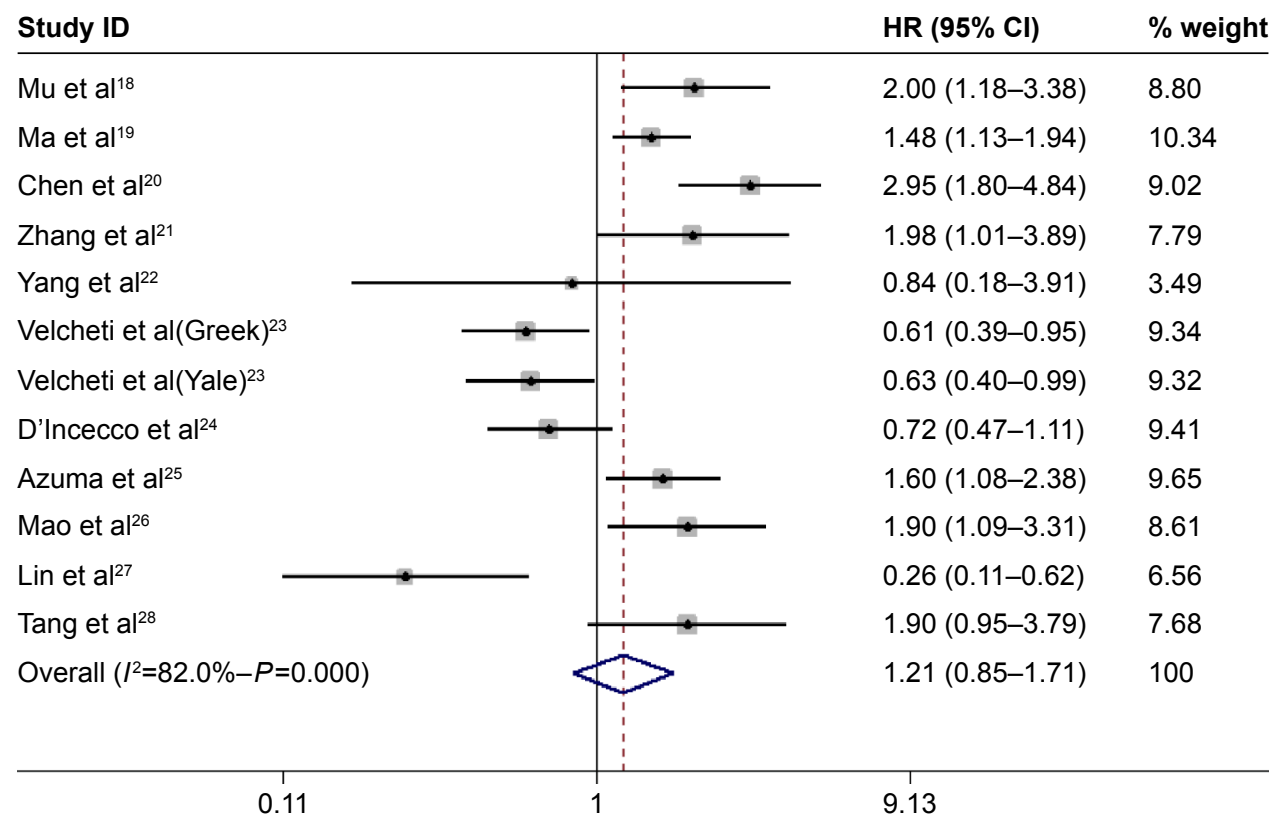

Figure 2 Forest plot showing HR from random-effects model for the association between PD-LI expression and OS.

Note: Weights are from random-effects analysis.

Abbreviations: $\mathrm{Cl}$, confidence interval; $\mathrm{HR}$, hazard ratio; OS, overall survival. 


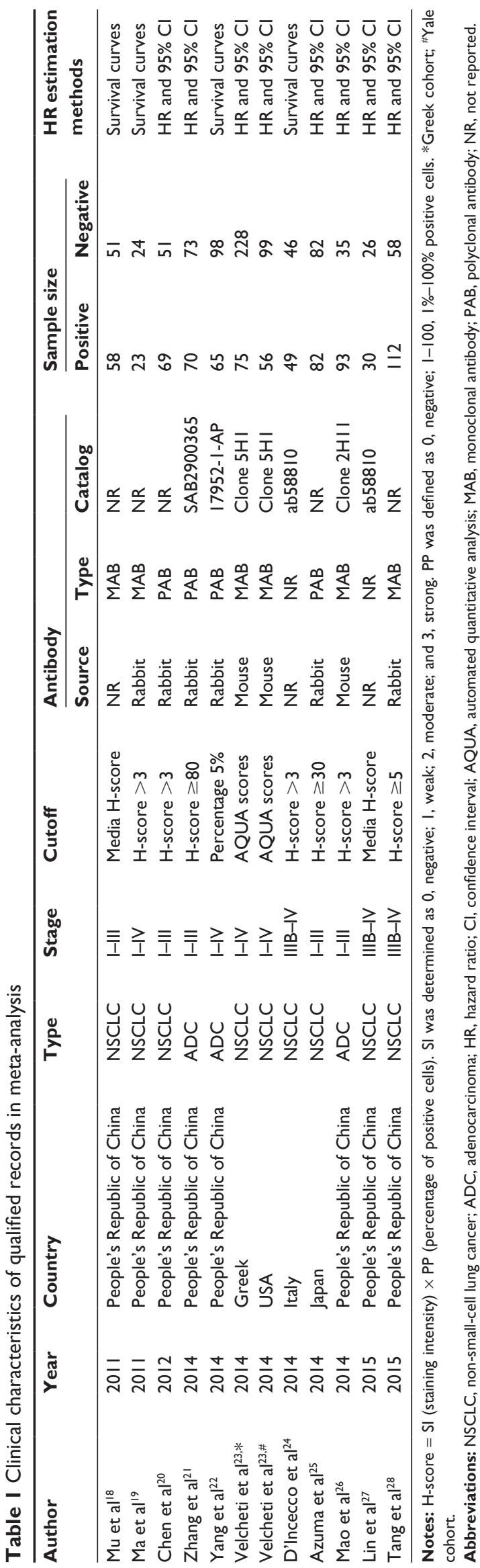

outcomes. The shape of the funnel plots did not indicate any obvious asymmetry (Figure 4).

\section{Discussion}

The aim of this meta-analysis was to explore the association between PD-L1 expression and the prognosis of patients with NSCLC. It was based on eleven eligible articles reporting on 12 studies with a total of 1,653 patients. The pooled data indicated no statistically significant difference between PD-L1 expression and prognosis in NSCLC. However, a subgroup analysis showed an association between PD-L1 expression and poor prognosis in studies conducted in the People's Republic of China. The prognostic role of PD-L1 expression in NSCLC has been evaluated extensively. Chen et $\mathrm{al}^{20}$ and Mao et $\mathrm{al}^{26}$ reported increased PD-L1 expression in NSCLC patients with poorly differentiated tumors and lymph node metastasis. High PD-L1 levels were shown to be associated with a significantly shorter OS. The relationship between PD-L1 expression and poor prognosis in NSCLC was confirmed in three other studies, ${ }^{18,19,21}$ whereas in another two studies, ${ }^{23,27}$ patients with NSCLC and higher PD-L1 expression had a better cumulated survival rate. In the remaining included studies, a significant correlation between PD-L1 expression and OS could not be established..$^{22,24,25,28}$ However, the differences in the definition of PD-L1-positivity/negativity and therapeutic regimen and the small sample sizes may have accounted for the contradictory results.

A potential association between PD-L1 expression and the prognosis of patients with NSCLC was assessed in previous meta-analyses. ${ }^{36-39}$ In three meta-analyses, it was found that NSCLC patients positive for PD-L1 expression had a poor OS. ${ }^{36,37,39}$ In another meta-analysis, PD-L1 was not a prognostic predictor for NSCLC..$^{38}$ However, the combined sample size of the three analyses was relatively small such that the results may not have been representative. Our metaanalysis enrolled nearly twice as many studies that consisted of nearly twice as many patients. The robustness of our results was established in subgroup and sensitivity analyses.

According to the subgroup analysis, high PD-L1 expression was a poor prognostic factor in Chinese but not in non-Chinese patients with NSCLC, which suggests that the association is dependent on race. In some studies, Chinese patients were pretreated with chemotherapy or surgery. However, some non-Chinese patients were also pretreated with these agents, and there have been no studies showing that these therapeutic agents affect the levels of PD-L1. The high PD-L1 expression in Chinese patients might be explained by the following: 1) three Chinese studies only 
Table 2 Subgroup analysis for the association between PD-LI expression and OS

\begin{tabular}{|c|c|c|c|c|c|c|c|}
\hline \multirow[t]{2}{*}{ Subgroup } & \multirow[t]{2}{*}{ No of study } & \multirow[t]{2}{*}{ No of patients } & \multirow[t]{2}{*}{ HR } & \multirow[t]{2}{*}{$95 \% \mathrm{Cl}$} & \multirow[t]{2}{*}{$P$-value } & \multicolumn{2}{|c|}{ Heterogeneity } \\
\hline & & & & & & $P$-value & $I^{2}, \%$ \\
\hline Overall & 12 & 1,653 & 1.21 & $0.85-|.7|$ & 0.29 & $<0.001$ & 82 \\
\hline \multicolumn{8}{|l|}{ Geographic region } \\
\hline People's Republic of China & 8 & 936 & 1.55 & $1.04-2.29$ & 0.03 & 0.001 & 72.5 \\
\hline Other than the People's Republic of China & 4 & 717 & 0.82 & $0.52-1.31$ & 0.41 & 0.003 & 79.0 \\
\hline \multicolumn{8}{|l|}{ Histological type (ADC \%) } \\
\hline$\geq 50$ & 9 & 1,170 & I.II & $0.77-1.62$ & 0.57 & $<0.001$ & 78.0 \\
\hline$<50$ & 3 & 569 & 1.52 & $0.58-4.03$ & 0.68 & $<0.001$ & 91.6 \\
\hline \multicolumn{8}{|l|}{ HR estimated } \\
\hline $\mathrm{HR} 95 \% \mathrm{Cl}$ & 8 & 1,239 & 1.19 & $0.72-1.98$ & 0.5 & $<0.001$ & 86.0 \\
\hline Kaplan-Meier curve & 4 & 414 & 1.24 & $0.76-2.01$ & 0.39 & 0.010 & 72.5 \\
\hline \multicolumn{8}{|l|}{ Antibody source } \\
\hline Mouse & 3 & 586 & 0.88 & $0.45-1.74$ & 0.30 & 0.003 & 83.1 \\
\hline Rabbit & 6 & 807 & 1.78 & $1.39-2.27$ & 0.03 & 0.222 & 28.4 \\
\hline NR & 3 & 260 & 0.76 & $0.28-2.06$ & 0.68 & $<0.001$ & 88.8 \\
\hline \multicolumn{8}{|l|}{ Antibody type } \\
\hline Monoclonal antibody & 6 & 912 & 1.24 & $0.80-1.91$ & 0.23 & $<0.001$ & 81.5 \\
\hline Polyclonal antibody & 4 & 590 & 1.97 & $1.34-2.89$ & 0.001 & 0.187 & 37.6 \\
\hline NR & 2 & 151 & 1.15 & $0.43-3.10$ & 0.78 & 0.013 & 83.7 \\
\hline
\end{tabular}

Notes: Bold values indicate PD-LI expression was significantly associated with poor prognosis in Chinese patients.

Abbreviations: PD-LI, programmed cell death-ligand I; ADC, adenocarcinomas; HR, hazard ratio; $\mathrm{Cl}$, confidence interval; OS, overall survival; NR, not reported.

included lung adenocarcinoma, and expression of PD-L1 increased in patients with these adenocarcinomas; and 2) the incidence of EGFR mutations was higher in East Asian patients than in Caucasian patients, because activation of the EGFR pathway induced PD-L1 expression. Thus, high PD-L1 expression may have existed.

There was also significant heterogeneity across the included studies. However, subgroup analyses of geographic region, cancer type, the methods used to estimate the HR, and the primary antibody used (source and type) failed to identify its source. Therefore, it is likely that the heterogeneity derived from differences in the baseline characteristics of the patients, the IHC methods used, the definition of positive/high PD-L1 expression, treatment, or the duration of follow-up. However, due to a lack of clinical information on these aspects, their contribution to the study heterogeneity could not be determined.

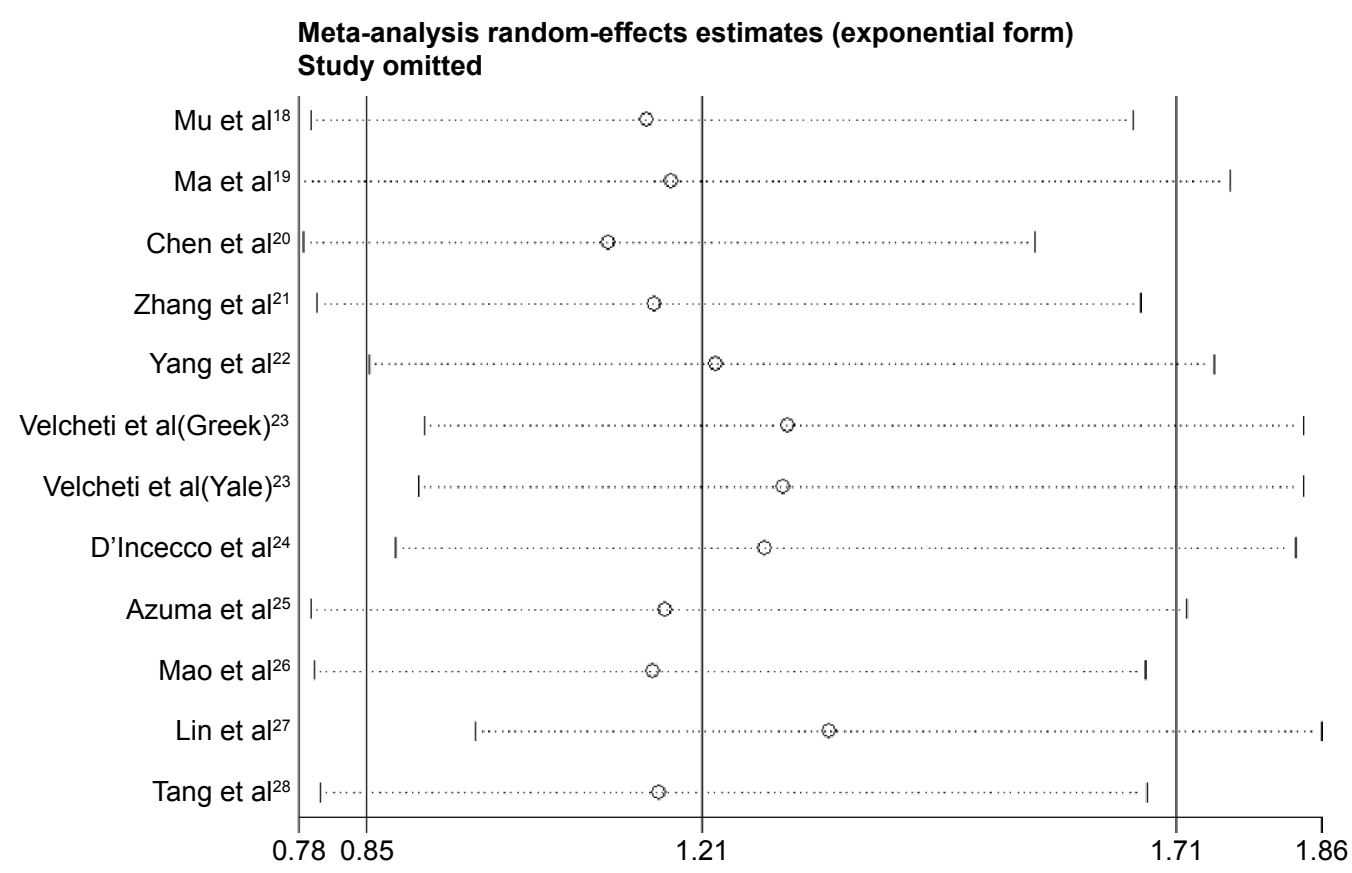

Figure 3 Sensitivity analysis via elimination of each study in turn. 


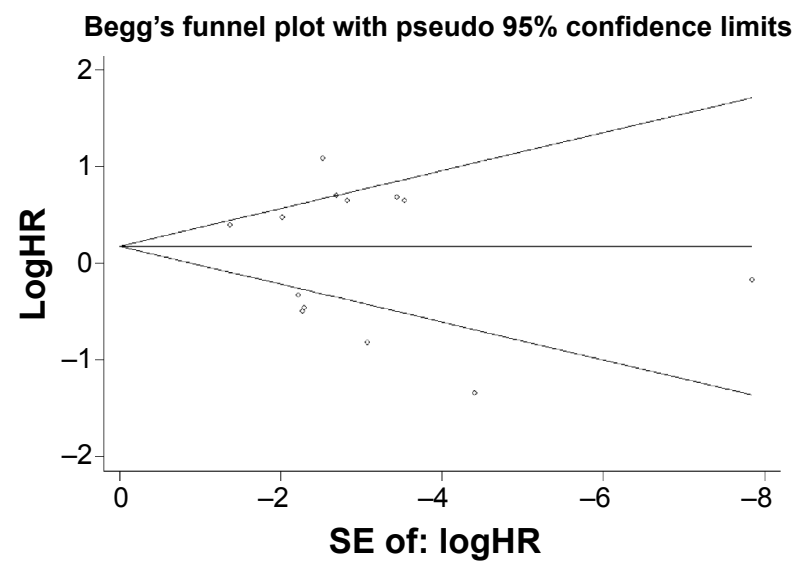

Figure 4 Funnel graph for assessment of potential publication bias in studies. Abbreviations: SE, standard error; HR, hazard ratio.

Regardless of these limitations, the results of the present study suggest that, in clinical trials, when anti-PD-L1 antibodies are used for cancer immunotherapy, patients with NSCLC should be carefully selected. Furthermore, PD-L1 is not a predictive biomarker for prognoses. Future studies involving the detection of PD-L1 expression in clinical applications should include larger sample sizes. Nonetheless, the present meta-analysis will provide useful guidelines for future studies. In the current meta-analysis, no publication bias was detected, and a sensitivity analysis showed that the conclusions were unchanged when any one study was omitted. Nonetheless, our meta-analysis had several limitations. First, both the pooled sample size and the sample size of each included study were relatively small. Despite pooling data from more than 1,500 patients with NSCLC, our study still lacked statistical power. Second, several HRs were extracted from Kaplan-Meier curves, which might have biased our results. Third, although all of the included studies detected PD-L1 expression using IHC, many different antibodies were used. The H-score was used to detect PD-L1 levels in eight studies, while the other studies used automated quantitative analysis. The cutoff values for positive PD-L1 expression also varied between studies. New studies using the same cutoff values are therefore needed for further confirmation. Fourth, despite several subgroup analyses, we were unable to identify the source of the between-study heterogeneity. Finally, some clinical parameters, such as sex, smoking status, histology, tumor stage, the presence or absence of lymph node metastasis, and tumor cell differentiation, may have had an effect on the prognosis of NSCLC patients. These were unavoidable limitations of the included studies; therefore, we could not adjust these confounding factors using statistical methods. In summary, our meta-analysis showed no statistically significant relationship between PD-L1 expression and the prognosis of patients with NSCLC. Additional, high-quality studies with larger sample sizes are needed to determine the prognostic value of PD-L1 expression in NSCLC.

\section{Acknowledgment}

This work was supported by the National Natural Science Foundation of China (Grant No: 81272610).

\section{Disclosure}

The authors report no conflicts of interest in this work.

\section{References}

1. Siegel R, Naishadham D, Jemal A. Cancer statistics, 2013. CA Cancer J Clin. 2013;63(1):11-30.

2. Siegel R, DeSantis C, Virgo K, et al. Cancer treatment and survivorship statistics, 2012. CA Cancer J Clin. 2012;62:220-241.

3. Molina JR, Yang P, Cassivi SD, Schild SE, Adjei AA. Non-small cell lung cancer: epidemiology, risk factors, treatment, and survivorship. Mayo Clin Proc. 2008;83(5):584-594.

4. Paesmans M, Sculier JP, Libert P, et al. Prognostic factors for survival in advanced non-small-cell lung cancer: univariate and multivariate analyses including recursive partitioning and amalgamation algorithms in 1,052 patients. The European Lung Cancer Working Party. J Clin Oncol. 1995;13(5):1221-1230.

5. Donnem T, Bremnes RM, Busund LT, Andersen S, Pezzella F. Gene expression assays as prognostic and predictive markers in early stage non-small cell lung cancer. J Thorac Dis. 2012;4(2):212-213.

6. Osarogiagbon RU. Predicting survival of patients with resectable non-small cell lung cancer: Beyond TNM. J Thorac Dis. 2012;4(2): 214-216.

7. Karachaliou N, Rosell R. Systemic treatment in EGFR-ALK NSCLC patients: second line therapy and beyond. Cancer Biol Med. 2014; 11(3):173-181

8. Knickelbein K, Zhang L. Mutant KRAS as a critical determinant of the therapeutic response of colorectal cancer. Genes Dis. 2014;2:4-12.

9. Sadeghi S, Olevsky O, Hurvitz SA. Profiling and targeting HER2positive breast cancer using trastuzumab emtansine. Pharmgenomics Pers Med. 2014;7:329-338.

10. Drake CG, Jaffee E, Pardoll DM. Mechanisms of immune evasion by tumors. Adv Immunol. 2006;90:51-81.

11. Agata Y, Kawasaki A, Nishimura H, et al. Expression of the PD-1 antigen on the surface of stimulated mouse T and B lymphocytes. Int Immunol. 1996;8(5):765-772.

12. Sznol M, Chen L. Antagonist antibodies to PD-1 and B7-H1 (PD-L1) in the treatment of advanced human cancer. Clin Cancer Res. 2013;19(5): 1021-1034.

13. Blank C, Brown I, Peterson AC, et al. PD-L1/B7-H1 inhibits the effector phase of tumor rejection by $\mathrm{T}$ cell receptor (TCR) transgenic CD8+ T cells. Cancer Res. 2004;64(3):1140-1145.

14. Haspot F, Fehr T, Gibbons C, et al. Peripheral deletional tolerance of alloreactive CD8 but not CD4 T cells is dependent on the PD-1/PD-L1 pathway. Blood. 2008;112(5):2149-2155.

15. Zou W, Chen L. Inhibitory B7-family molecules in the tumor microenvironment. Nat Rev Immunol. 2008;8(6):467-477.

16. Mischinger J, Froehlich E, Griesbacher A, et al. Prognostic relevance of $\mathrm{B} 7 \mathrm{H} 1$ and $\mathrm{B} 7 \mathrm{H} 3$ protein expressions in metastatic clear cell renal cell carcinoma. J Clin Oncol. 2010;28(Suppl):abstract e150674.

17. Taube JM, Anders RA, Young GD, et al. Colocalization of inflammatory response with B7-h1 expression in human melanocytic lesions supports an adaptive resistance mechanism of immune escape. Sci Transl Med. 2012;4(127):127-137. 
18. Mu CY, Huang JA, Chen Y, Chen C, Zhang XG. High expression of PD-L1 in lung cancer may contribute to poor prognosis and tumor cells immune escape through suppressing tumor infiltrating dendritic cells maturation. Med Oncol. 2011;28(3):682-688.

19. Ma W, Luo DZ, Chen Y, Dang YW. Expression and clinical significance of PD-L1 and PD-1 in non-small cell lung cancer. J Pract Med. 2011;27:1551-1554.

20. Chen YB, Mu CY, Huang JA. Clinical significance of programmed death-1 ligand-1 expression in patients with non-small cell lung cancer: a 5-year-follow-up study. Tumori. 2012;98(6):751-755.

21. Zhang Y, Wang L, Li Y, et al. Protein expression of programmed death 1 ligand 1 and ligand 2 independently predict poor prognosis in surgically resected lung adenocarcinoma. Onco Targets Ther. 2014;7:567-573.

22. Yang CY, Lin MW, Chang YL, Wu CT, Yang PC. Programmed cell death-ligand 1 expression in surgically resected stage I pulmonary adenocarcinoma and its correlation with driver mutations and clinical outcomes. Eur J Cancer. 2014;50(7):1361-1369.

23. Velcheti V, Schalper KA, Carvajal DE, et al. Programmed death ligand-1 expression in non-small cell lung cancer. Lab Invest. 2014; 94(1):107-116.

24. D'Incecco A, Andreozzi M, Ludovini V, et al. PD-1 and PD-L1 expression in molecularly selected non-small-cell lung cancer patients. $\mathrm{Br}$ J Cancer. 2015;112(1):95-102.

25. Azuma K, Ota K, Kawahara A, et al. Association of PD-L1 overexpression with activating EGFR mutations in surgically resected nonsmallcell lung cancer. Ann Oncol. 2014;25(10):1935-1940.

26. Mao Y, Li W, Chen K, et al. B7-H1 and B7-H3 are independent predictors of poor prognosis in patients with non-small cell lung cancer. Oncotarget. 2015;6(5):3452-3461.

27. Lin C, Chen X, Li M, et al. Programmed death-ligand 1 expression predicts tyrosine kinase inhibitor response and better prognosis in a cohort of patients with epidermal growth factor receptor mutation-positive lung adenocarcinoma. Clin Lung Cancer. 2015;16(5):e25-e35

28. Tang Y, Fang W, Zhang Y, et al. The association between PD-L1 and EGFR status and the prognostic value of PD-L1 in advanced nonsmall cell lung cancer patients treated with EGFR-TKIs. Oncotarget. 2015;6(16):14209-14219.
29. Moher D, Liberati A, Tetzlaff J, Altman DG; PRISMA Group. Preferred reporting items for systematic reviews and meta-analyses: the PRISMA statement. J Clin Epidemiol. 2009;62(10):1006-1012.

30. Tierney JF, Stewart LA, Ghersi D, Burdett S, Sydes MR. Practical methods for incorporating summary time-to-event data into meta-analysis. Trials. 2007;8:16.

31. Mantel N, Haenszel W. Statistical aspects of the analysis of data from retrospective studies of disease. J Natl Cancer Inst. 1959;22(4): 719-748.

32. DerSimonian R, Laird N. Meta-analysis in clinical trials. Control Clin Trials. 1986;7(3):177-188.

33. Ylmaz M, Bayazit YA, Ciftci TU, et al. Association of serotonin transporter gene polymorphism with obstructive sleep apnea syndrome. Laryngoscope. 2005;115(5):832-836.

34. Begg CB, Mazumdar M. Operating characteristics of a rank correlation test for publication bias. Biometrics. 1994;50(4):1088-1101.

35. Egger M, Davey SG, Schneider M, Minder C. Bias in meta-analysis detected by a simple, graphical test. BMJ. 1997;315(7109):629-634.

36. Zhou ZJ, Zhan P, Song Y. PD-L1 over-expression and survival in patients with non-small cell lung cancer: a meta-analysis. Transl Lung Cancer Res. 2015;4(2):203-208.

37. Wang A, Wang HY, Liu Y, et al. The prognostic value of PD-L1 expression for non-small cell lung cancer patients: a meta-analysis. Eur J Surg Oncol. 2015;41(4):450-456.

38. Zhang Y, Kang S, Shen J, et al. Prognostic significance of programmed cell death 1 (PD-1) or PD-1 ligand 1 (PD-L1) expression in epithelial-originated cancer: a meta-analysis. Medicine (Baltimore). 2015;94(6):e515.

39. Pan ZK, Ye F, Wu X, An HX, Wu JX. Clinicopathological and prognostic significance of programmed cell death ligand 1 (PD-L1) expression in patients with non-small cell lung cancer: a meta-analysis. J Thorac Dis 2015;7(3):462-470.
OncoTargets and Therapy

\section{Publish your work in this journal}

OncoTargets and Therapy is an international, peer-reviewed, open access journal focusing on the pathological basis of all cancers, potential targets for therapy and treatment protocols employed to improve the management of cancer patients. The journal also focuses on the impact of management programs and new therapeutic agents and protocols on

\section{Dovepress}

patient perspectives such as quality of life, adherence and satisfaction. The manuscript management system is completely online and includes a very quick and fair peer-review system, which is all easy to use. Visit http://www.dovepress.com/testimonials.php to read real quotes from published authors. 